\title{
APPROACH REGIONS FOR THE SQUARE ROOT OF THE POISSON KERNEL AND BOUNDED FUNCTIONS
}

\author{
P. SJÖGREN
}

If the Poisson kernel of the unit disc is replaced by its square root, it is known that normalised Poisson integrals of $L^{p}$ boundary functions converge almost everywhere at the boundary, along approach regions wider than the ordinary nontangential cones. The sharp approach region, defined by means of a monotone function, increases with $p$. We make this picture complete by determining along which approach regions one has almost everywhere convergence for $L^{\infty}$ boundary functions.

\section{INTRODUCTION}

Let

$$
P(z, \theta)=\frac{1}{2 \pi} \frac{1-|z|^{2}}{\left|z-e^{i \theta}\right|^{2}}
$$

be the Poisson kernel of the unit disc $U \subset \mathbb{C}$. With $\mathbb{T}=\partial U=\mathbb{R} / 2 \pi$, the Poisson integral of a function $f \in L^{1}(\mathbb{T})$ is $P f(z)=\int_{\mathbb{T}} P(z, \theta) f(\theta) d \theta$.

It is well known that the harmonic function $P f(z)$ converges to $f(\theta)$ for almost all $\theta \in \mathbb{T}$ as $z \rightarrow e^{i \theta}$ and $z$ stays in the nontangential cone which one can define by the inequality $|\arg z-\theta|<$ constant $(1-|z|)$. Moreover, these cones are best (that is, largest) possible if one considers regions of the type $|\arg z-\theta|<h(1-|z|)$ for increasing functions $h$. The situation is the same for $L^{p}$ boundary functions $f$, for $1 \leqslant p \leqslant \infty$.

However, if we use the square root of $P$, the convergence properties are different. Define $P_{0} f(z)=\int \sqrt{P(z, \theta)} f(\theta) d \theta$. Then $P_{0} f$ is an eigenfunction of the hyperbolic Laplacian. This and other powers of the Poisson kernel are used in connection with representations of the group $S L(2, \mathbb{R})$. To get boundary convergence, one must normalise $P_{0} f$ by setting

$$
\mathcal{P}_{0} f(z)=\frac{P_{0} f(z)}{P_{0} 1(z)} .
$$

Here 1 denotes the constant boundary function.

Received 18th December, 1996

Copyright Clearance Centre, Inc. Serial-fee code: 0004-9729/97 \$A2.00+0.00. 
First of all, $\mathcal{P}_{0} f(z)$ converges for continuous $f$; indeed $\mathcal{P}_{0} f$ can then be extended by $f$ to a continuous function in $\bar{U}$. This is because the operator $\mathcal{P}_{0}$ is given by convolution with an approximate identity in $\mathbb{T}$. If $f \in L^{p}(\mathbb{T})$ and $1 \leqslant p<\infty$, it is known that $\mathcal{P}_{0} f(z)$ converges to $f(\theta)$ for almost all $\theta \in \mathbb{T}$ as $z \rightarrow e^{i \theta}$ and $z$ stays in the approach region

$$
R_{p}(\theta)=\left\{z \in U:|\arg z-\theta|<C(1-|z|)\left(\log \frac{1}{1-|z|}\right)^{p}\right\} .
$$

Here $C<\infty$ is arbitrary. This result was proved for $p=1$ by the author [5] and for $1<p<\infty$ by Rönning [2]. Clearly $\overline{R_{p}(\theta)}$ intersects $\mathbb{T}$ only at $e^{i \theta}$. Notice that $R_{p}(\theta)$ is wider than the nontangential cones near $e^{i \theta}$, and that $R_{p}(\theta)$ increases with $p$. Further results for the square root of the Poisson kernel in more general settings can be found in Rönning [3] and [4] and in Sjögren [6].

We point out that one also has convergence, after normalisation, if any power larger than $1 / 2$ of the Poisson kernel is used. But then the approach regions are like those of the Poisson kernel itself. Only the square root is different.

For $\mathcal{P}_{0} f$ with $f \in L^{\infty}(\mathbb{T})$, it trivially follows that one has, almost everywhere, convergence along $R_{p}(\theta)$ for any $p<\infty$. The object of this note is to show that one can have wider approach regions here. We determine precisely which regions, defined by means of an increasing function, are admissible for $L^{\infty}$.

THEOREM. The following are equivalent for any increasing function $h: \mathbb{R}_{+} \rightarrow \mathbb{R}_{+}$.

(i) For any $f \in L^{\infty}(\mathbb{T})$ one has for almost all $\theta \in \mathbb{T}$

$$
\mathcal{P}_{0} f(z) \rightarrow f(\theta) \text { as } z \rightarrow e^{i \theta} \text { and }|\arg z-\theta|<h(1-|z|) .
$$

(ii) $h(t)=O\left(t^{1-\varepsilon}\right)$ as $t \rightarrow 0$, for any $\varepsilon>0$.

Here $O$ can be replaced by $o$, since $\varepsilon$ can be varied.

Essentially all proofs of almost everywhere convergence results for various Poisson integrals of $L^{p}$ functions, $p<\infty$, use a maximal function argument. The starting point of such an argument is already established convergence for continuous or smooth functions. The maximal function corresponding to a certain kind of convergence is defined (essentially) by replacing lim by sup, taken over the approach region, and by replacing the function by its absolute value. From a strong or weak type $(p, p)$ estimate for the maximal operator, almost everywhere convergence then follows, via approximation with smooth functions.

But this argument breaks down for $L^{\infty}$, since the continuous functions do not form a dense subspace of $L^{\infty}$. To prove the above theorem, we shall instead use a recent 
result due to Bellow and Jones [1]. It states that almost everywhere convergence follows if the restriction of the maximal operator to the unit ball in $L^{\infty}$ is continuous at 0 for the topology of convergence in measure.

By $C$ we denote various (large) positive constants, and $F \sim G$ means $1 / C \leqslant$ $F / G \leqslant C$.

\section{Proof of THE THEOREM}

Write $t=1-|z|$ and thus $z=(1-t) e^{i \theta}$. Then

$$
\mathcal{P}_{0} f(z)=R_{t} * f(\theta)
$$

where the convolution is taken in $\mathbb{T}$ and

$$
R_{t}(\theta)=\frac{1}{\sqrt{2 \pi}} \frac{\sqrt{t(2-t)}}{\left|(1-t) e^{i \theta}-1\right|} \frac{1}{P_{0} 1(1-t)}
$$

Here $\theta \in \mathbb{T}=(-\pi, \pi]$. Since $P_{0} 1(1-t) \sim \sqrt{t} \log 1 / t$, the order of magnitude of $R_{t}$ is given by

$$
R_{t}(\theta) \sim Q_{t}(\theta)=\frac{1}{\log 1 / t} \frac{1}{t+|\theta|}
$$

Here and in the sequel, we assume $t<1 / 2$, that is, we disregard the disc $|z| \leqslant 1 / 2$.

Write $\tau_{\eta} f(\theta)=f(\theta-\eta)$. Then the convergence in (i) means

$$
\tau_{\eta} R_{t} * f(\theta) \rightarrow f(\theta), \quad t \rightarrow 0, \quad|\eta|<h(t) .
$$

The relevant maximal function for our problem is

$$
\left.M_{0} f(\theta)=\sup _{|\operatorname{larg} z-\theta|<h(1-|z|)}|z|>1 / 2\right\}
$$

Notice that $M_{0} f(\theta)$ is dominated by a constant times

$$
M f(\theta)=\sup _{\substack{|\eta|<h(t) \\ 0<t<1 / 2}} \tau_{\eta} Q_{t} *|f|(\theta)
$$

Proposition. Assume that (ii) holds, and let $\varepsilon>0$. Given $\delta>0$, there exists $\kappa>0$ such that for $f \in L^{\infty}(\mathbb{T})$ with $\|f\|_{\infty} \leqslant 1$

$$
|\{\theta \in \mathbb{T}: M f(\theta)>\varepsilon\}|<\delta
$$


if $\|f\|_{1}<\kappa$.

Here $\|\cdot\|_{p}$ is the norm in $L^{p}(\mathbb{T})$, and $|\cdot|$ is Lebesgue measure in $\mathbb{T}$. This proposition means that $M$ is continuous at 0 for the topology of convergence in measure, when restricted to the unit ball in $L^{\infty}$. For this, see [1, p.158]. We can then apply Corollary 1 of [1] to the family of operators $f \rightarrow \tau_{\eta} R_{t} * f,|\eta|<h(t), 0<t<1 / 2$. This is not a sequence of operators, but one can easily either extend Bellow and Jones's result to larger families or reduce our convergence problem to a sequence. Thus the implication (ii) $\Rightarrow$ (i) is a consequence of the proposition.

Proof of the Proposition: We can clearly assume that $\varepsilon$ is small and that $f \geqslant 0$.

Consider first $Q_{t} * f$ with $t$ large in the sense that $e^{-4 / \varepsilon} \leqslant t<1 / 2$. Observe that $Q_{t} \leqslant(t \log 1 / t)^{-1}$ in $\mathbb{T}$, and that the function $t \rightarrow t \log 1 / t$ is increasing near 0 . Thus $Q_{t} * f \leqslant e^{4 / \varepsilon} \varepsilon / 4\|f\|_{1}$ in $\mathbb{T}$, so that $Q_{t} * f<\varepsilon$ if $\|f\|_{1}<e^{-4 / \varepsilon}$. This means that in the supremum defining $M f$, we can assume that $t<e^{-4 / \varepsilon}$.

Write

$$
Q_{t}(\theta)=Q_{t}(\theta) \chi_{|\theta| \leqslant 2 h(t)}+Q_{t}(\theta) \chi_{2 h(t)<|\theta|}=Q_{t}^{1}(\theta)+Q_{t}^{2}(\theta)
$$

Letting

$$
M_{j} f(\theta)=\sup _{\substack{|\eta|<h(t) \\ 0<t<e^{-4 / \varepsilon}}} \tau_{\eta} Q_{t}^{j} * f(\theta), \quad j=1,2,
$$

we get $M f \leqslant M_{1} f+M_{2} f$ and thus

$$
\{M f>\varepsilon\} \subset\left\{M_{1} f>\varepsilon / 2\right\} \cup\left\{M_{2} f>\varepsilon / 2\right\} .
$$

To deal with $M_{2} f$, observe that when $|\eta|<h(t)$

$$
\tau_{\eta} Q_{t}^{2}(\theta) \leqslant \frac{1}{\log 1 / t} \frac{1}{t+|\theta-\eta|} \chi|\theta-\eta|>2 h(t) \leqslant \frac{2}{\log 1 / t} \frac{1}{t+|\theta|} .
$$

The last expression is a decreasing function of $|\theta|$, whose integral in $\mathbb{T}$ is bounded uniformly in $t$. It is well known that convolution by such a function is controlled by the Hardy-Littlewood maximal operator $M_{H L}$, so that $M_{2} f \leqslant C M_{\mathrm{HL}} f$. Since $M_{\mathrm{HL}}$ is of weak type $(1,1)$, we obtain

$$
\left|\left\{M_{2} f>\varepsilon / 2\right\}\right| \leqslant C \varepsilon^{-1}\|f\|_{1} .
$$

Finally, we consider $M_{1} f$. If $M_{1} f(\theta)>\varepsilon / 2$, there exist $t \in\left(0, e^{-4 / \varepsilon}\right)$ and $\eta$ with $|\eta| \leqslant h(t)$ such that $Q_{t}^{1} * f(\theta-\eta)>\varepsilon / 2$. This means that

$$
\int_{|\varphi|<2 h(t)} \frac{1}{\log 1 / t} \frac{1}{t+|\varphi|} f(\theta-\eta-\varphi) d \varphi>\frac{\varepsilon}{2} \text {. }
$$


Clearly only the values of $f$ in the interval

$$
[\theta-\eta-2 h(t), \theta-\eta+2 h(t)] \subset[\theta-3 h(t), \theta+3 h(t)]
$$

matter here. We shall compare the integral in (3) with

$$
m=\int_{\theta-3 h(t)}^{\theta+3 h(t)} f(\varphi) d \varphi
$$

Our claim is

$$
\int_{-2 h(t)}^{2 h(t)} \frac{1}{t+|\varphi|} f(\theta-\eta-\varphi) d \varphi \leqslant 2 \log \frac{t+m / 2}{t}
$$

This actually means that, with the restrictions $0 \leqslant f \leqslant 1$ and $\int f=m$, the integral in (4) is maximal when $f$ is given by $f(\theta-\eta-\varphi)=\chi_{[-m / 2, m / 2]}(\varphi)$. To obtain (4), observe first that

$$
\frac{1}{t+|\varphi|} \chi_{[-2 h(t), 2 h(t)]}(\varphi)=\int \chi_{[-\tau, \tau]}(\varphi) d \mu(\tau),
$$

where $\mu$ is the positive measure

$$
d \mu(\tau)=(t+\tau)^{-2} \chi_{[0,2 h(t)]} d \tau+(t+2 h(t))^{-1} \delta_{2 h(t)} .
$$

From Fubini's theorem, we then have

$$
\int_{-2 h(t)}^{2 h(t)} \frac{1}{t+|\varphi|} f(\theta-\eta-\varphi) d \varphi=\int d \mu(\tau) \int \chi_{[-\tau, \tau]}(\varphi) f(\theta-\eta-\varphi) d \varphi .
$$

For each $\tau$, it is trivial to see that the inner integral here is maximal when $f(\theta-\eta-\varphi)=$ $\chi_{[-m / 2, m / 2]}(\varphi)$. Applying again Fubini's theorem, we conclude

$$
\int_{-2 h(t)}^{2 h(t)} \frac{1}{t+|\varphi|} f(\theta-\eta-\varphi) d \varphi \leqslant \int \frac{1}{t+|\varphi|} \chi_{[-m / 2, m / 2]}(\varphi) d \varphi=2 \log \frac{t+m / 2}{t},
$$

and (4) follows.

Combining (3) and (4), we have

$$
\frac{2}{\log 1 / t} \log \frac{t+m / 2}{t}>\frac{\varepsilon}{2}
$$

Hence $\log (t+m / 2) / t>\log t^{-\varepsilon / 4}$, and so $m / 2 t>t^{-\varepsilon / 4}-1$. But $t^{-\varepsilon / 4}>2$ because of our assumption $t<e^{-4 / \varepsilon}$. This implies $m / 2 t>t^{-\varepsilon / 4} / 2$ and finally $m>t^{1-\varepsilon / 4}$. 
To sum up, we have shown that for each $\theta$ with $M_{1} f(\theta)>\varepsilon / 2$ there exists a $t=t(\theta)$ such that the interval $J(\theta)=[\theta-3 h(t), \theta+3 h(t)]$ has the property

$$
\int_{J(\theta)} f(\varphi) d \varphi>t^{1-\varepsilon / 4}
$$

A standard covering argument then produces a sequence $\left(\theta_{i}, t_{i}\right)$ with $M_{1} f\left(\theta_{i}\right)>\varepsilon / 2$ and $t_{i}=t\left(\theta_{i}\right)$ such that the corresponding $J\left(\theta_{i}\right)$ are disjoint and the scaled intervals $J^{\prime}\left(\theta_{i}\right)=\left[\theta_{i}-9 h\left(t_{i}\right), \theta_{i}+9 h\left(t_{i}\right)\right]$ cover the set $\left\{M_{1} f>\varepsilon / 2\right\}$. In particular, we get

$$
\|f\|_{1} \geqslant \sum_{i} \int_{J\left(\theta_{i}\right)} f(\varphi) d \varphi \geqslant \sum_{i} t_{i}^{1-\varepsilon / 4}
$$

Our hypothesis (ii) implies that $h(t) \leqslant C t^{1-\varepsilon / 4}$ for some $C$ depending on $\varepsilon$, and so

$$
\left|\left\{M_{1} f>\varepsilon / 2\right\}\right| \leqslant \sum_{i}\left|J^{\prime}\left(\theta_{i}\right)\right| \leqslant 18 \sum_{i} h\left(t_{i}\right) \leqslant C \sum_{i} t_{i}^{1-\varepsilon / 4} \leqslant C\|f\|_{1}
$$

in view of (5).

Combining this with (1) and (2), we get

$$
|\{M f>\varepsilon\}| \leqslant C\|f\|_{1}, \quad C=C(\varepsilon) .
$$

The proposition follows.

It remains to prove that (i) implies (ii), and we assume that (ii) is false. Then there exist $\varepsilon \in(0,1)$ and a sequence $t_{i} \rightarrow 0$ such that $h\left(t_{i}\right) / t_{i}^{1-\varepsilon} \rightarrow \infty$. We can assume that $\sum_{i} t_{i}^{1-\varepsilon} / h\left(t_{i}\right)<\infty$. Let $E_{i} \subset \mathbb{T}$ be the union of at most $C / h\left(t_{i}\right)$ intervals of length $t_{i}^{1-\varepsilon}$, chosen in such a way that the distance from any point of $\mathbb{T}$ to $E_{i}$ is at most $h\left(t_{i}\right)$. If $\theta \in \partial E_{i}$, it is clear that

$$
\mathcal{P}_{0} \chi E_{i}\left(\left(1-t_{i}\right) e^{i \theta}\right) \geqslant \frac{1}{C \log 1 / t_{i}} \int_{0}^{t_{i}^{1-\varepsilon}} \frac{d \varphi}{t_{i}+\varphi} \geqslant \frac{1}{C \log 1 / t_{i}} \int_{t_{i}}^{t_{i}^{1-\varepsilon}} \frac{d \varphi}{\varphi}=\varepsilon / C .
$$

Thus for any $\theta \in \mathbb{T}$ one has

$$
\sup _{|\eta|<h\left(t_{i}\right)} \mathcal{P}_{0} \chi_{E_{i}}\left(\left(1-t_{i}\right) e^{i(\theta-\eta)}\right) \geqslant \varepsilon / C .
$$

Since $\left|E_{i}\right| \leqslant C t_{i}^{1-\varepsilon} / h\left(t_{i}\right)$, we can choose $i_{0}$ so large that the measure $|E|$ of the set $E=\bigcup_{i>i_{0}} E_{i}$ is arbitrarily small. But clearly

$$
\varlimsup_{\substack{t \rightarrow 0 \\|\eta|<h(t)}} \mathcal{P}_{0} \chi_{E}\left((1-t) e^{i(\theta-\eta)}\right) \geqslant \varepsilon / C
$$

for each $\theta$. Thus we do not have almost everywhere convergence to $\chi_{E}$ along the region defined by $h$, and (i) is disproved. This completes the proof of the theorem. 


\section{REFERENCES}

[1] A. Bellow and R. L. Jones, 'A Banach principle for $L^{\infty}$, Adv. Math. 120 (1996), 155-172.

[2] J.-O. Rönning, 'Convergence for square roots of the Poisson kernel in weakly tangential regions', Math. Scand. (to appear).

[3] J.-O. Rönning, 'A convergence result for square roots of the Poisson kernel in the bidisk', Preprint 1993-34. Dept. of Math., Chalmers Univ. of Techn. and Univ. of Göteborg.

[4] J.-O. Rönning, 'On convergence for the square root of the Poisson kernel in symmetric spaces of rank 1', Studia Math. (to appear).

[5] P. Sjögren, 'Une remarque sur la convergence des fonctions propres du Laplacien à valeur propre critique', in Théorie du Potentiel, Proceedings Orsay 1983, Lecture Notes in Mathematics 1096 (Springer-Verlag, 1984), pp. 141-147.

[6] P. Sjögren, 'Convergence for the square root of the Poisson kernel', Pacific J. Math. 131 (1988), 361-391.

Department of Mathematics

Chalmers University of Technology and Göteborg University

S-412 96 Göteborg

Sweden

email: peters@math.chalmers.se 